\title{
Comparison of blood flow patterns in cerebral aneurysms
}

\author{
K. Shimano, T. Kudo \& Y. Enomoto \\ Department of Mechanical Systems Engineering, \\ Musashi Institute of Technology, Japan
}

\begin{abstract}
Thrombogenesis is said to play an important role in the rupture of cerebral artery aneurysms and it was reported that the degree of platelet aggregation in an aneurysm had a significant correlation with the flow pattern in the aneurysmal dome. In this study, flows in three different models of cerebral saccular aneurysms at artery bifurcations were numerically investigated to compare flow patterns from a viewpoint of likelihood of platelet aggregation. It was shown that the relative size of the aneurysmal dome had a greater influence on the formation of a low-speed region responsible for active platelet aggregation than geometric features such as the aspect ratio of the aneurysm and the angle of the bifurcation.

Keywords: cerebral saccular aneurysm, computational fluid dynamics, platelet aggregation, anterior communicating artery, artery bifurcation.
\end{abstract}

\section{Introduction}

Rupture of a cerebral artery aneurysm causes a life-threatening subarachnoid haemorrhage. Although not all aneurysms rupture, it is difficult to predict which aneurysms are likely to do so. Ujiie et al. [1] reported a significant correlation between the probability of rupture and aspect ratio of the aneurysmal dome depth to the neck width: almost $80 \%$ of ruptured aneurysms had aspect ratio larger than 1.6. According to a theory of Ujiie et al. [2], thrombogenesis is likely in an aneurysm of high aspect ratio due to the extremely slow blood flow in it. Ensuing fibrinolysis in which thrombi are dissolved is considered to cause damage to the endothelium. Takahashi et al. [3] showed evidence supporting the hypothesis: a canine blood flow in endothelialised model aneurysms made of glass was visualised and thrombogenesis was observed for a large aspect ratio. 
For further progress in the area of the rupture prediction, more understanding of the flow and the thrombogenesis in aneurysms is crucial. Approaches based on numerical techniques such as computational fluid dynamics (CFD) are promising for this purpose. In the previous study [4], the authors' group applied an unsteady Navier-Stokes solver to the flow in one of model aneurysms tested by Takahashi et al. Furthermore, the authors proposed a platelet aggregation model which mathematically describes a series of physiological reactions of platelets to adenosine diphosphate (ADP). When the aggregation model was combined with CFD, the computational results showed that a lot of platelets were aggregated along the aneurysmal wall where the blood flow was considerably slow. It was also demonstrated that the flow pattern in the aneurysmal dome was closely correlated with the location of platelet aggregation. Therefore, it is important to classify blood flow patterns into some groups by investigation of a number of different aneurysms. Although a lot of CFD applications to blood flows in cerebral aneurysms have been reported (for example, References [5] and [6]), blood flow patterns were not discussed from a viewpoint of platelet aggregation.

In this study, unsteady blood flow simulations with unstructured grids were conducted for three different model aneurysms in order to compare flow patterns in terms of likelihood of platelet aggregation.

\section{Model aneurysms}

Three aneurysms at artery bifurcations were modelled for the flow simulation because it is known that approximately $50 \%$ of cerebral aneurysms are found at bifurcations where anterior communicating arteries separate from anterior cerebral arteries. Each model had an aspect ratio larger than 1.6 so that aneurysms with potential risk of rupture could be investigated.

Model 1 is shown in fig.1.The aneurysmal dome was located at a Y-shaped bifurcation and the whole geometry was symmetrical about the centre plane, which is, in fig.1, parallel to the surface of the page. Model 1 was based on one of the glass aneurysms made and experimentally tested by Takahashi et al [3]. Also Shimano et al. [4] used this glass model for application of the aggregation model. The geometric data was obtained manually from several digital images of the glass aneurysm cut in half because the original glass model was a handcraft and no CAD data was available. The depth of the aneurysmal dome and the neck width measured $6.5 \mathrm{~mm}$ and $2.8 \mathrm{~mm}$, respectively, with 2.3 aspect ratio. The centre line of each tube lay on the same plane identical to the plane of symmetry. A thick arrow in fig. 1 indicates the direction of the inlet flow. The diameter of the inlet tube was $2 \mathrm{~mm}$. A curve of the cyclically changing flow rate, which had been recorded during the experiment by Takahashi et al., was imposed as the inlet boundary conditions. The period of the cyclic change was $0.276 \mathrm{sec}$. and the Womersley parameter was 2.39. The Reynolds number at the inlet ranged between 244 and 485 . At the bifurcation, $40 \%$ of the blood proceeded to the anterior communicating artery and the rest continued flowing in the anterior cerebral artery. This ratio of the flow rates remained the same regardless of time. 
Model 2 was a scale-up model originally designed by Shimamura [7] who had visualised the flow pattern in the dome. As shown in fig.2, the model had an aneurysmal dome at a $\mathrm{T}$-shaped cerebral bifurcation where two arteries met at the right angle. The whole model geometry was symmetrical about the centre plane in the same manner as Model 1. The main pipe (anterior cerebral artery) and the branch (anterior communicating artery) were $5 \mathrm{~mm}$ and $3 \mathrm{~mm}$ in diameter, respectively.

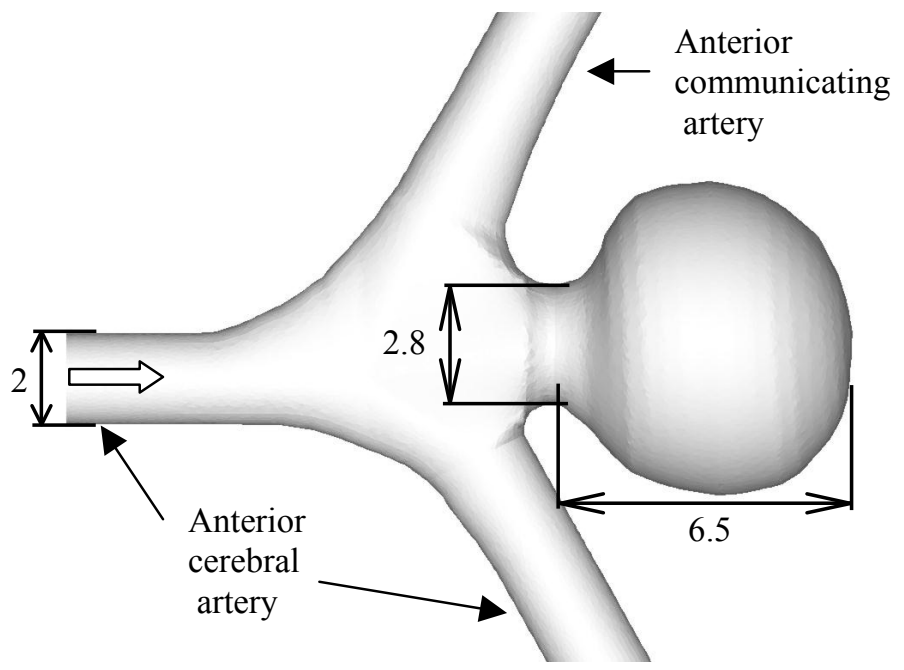

Figure 1: $\quad$ The geometry of Model 1 (all dimensions in $\mathrm{mm}$ ).

In Model 2, the dome was an ellipsoid with the major and minor axes of 8 and $4 \mathrm{~mm}$ and inclined by 45 degrees. It is difficult to define the depth of the dome or the neck width because the dome was partly embedded into the arteries. However, the length of the major axis, $8 \mathrm{~mm}$, seems a good approximation to the dome depth and, similarly, the minor axis can be used to evaluate the neck width. Using these approximations, the aspect ratio was determined to be 2 .

As a sinusoidal curve had been used for the inlet flow rate in Shimamura's experiment, the same curve was adopted as the inlet boundary conditions. The Womersley parameter, min. and max. Reynolds numbers were 2.4, 399 and 797, respectively, which represented a flow in a real cerebrovascular. Throughout the cycle, $26 \%$ of the incoming blood moved to the branch at the bifurcation.

Influences of the bifurcation angle could be investigated by comparing Models 1 and 2. However, in these two models, the centre line of each pipe lay on the same plane and this is uncommon for real cerebral bifurcations. Therefore, the authors used another model with more realistic features. Model 3, shown in fig.3, consisted of 3-dimensionally curved pipes and an ellipsoidal aneurysm. The direction of protrusion of the dome was determined according to the most likely features of anterior communicating artery aneurysms mentioned in Reference [8]. In Model 3, the main pipe (anterior cerebral artery) shrank a little: the diameter measured $3 \mathrm{~mm}$ near the inlet and $2.7 \mathrm{~mm}$ after the bifurcation. On 
the other hand, the branch (anterior communicating artery) was $1.8 \mathrm{~mm}$ in diameter. The dome was an ellipsoid with the major and minor axes of 4 and 2 $\mathrm{mm}$. The aspect ratio was determined to be 2 in the same manner as in Model 2.

A flow rate curve similar to that used for Model 1 was imposed as the inlet boundary conditions. The Womersley parameter was 2.25 . The maximum and minimum Reynolds numbers at the inlet were 366 and 728 , respectively. $30 \%$ of the blood flowed into the anterior communicating artery.

\section{Computational techniques}

The unsteady incompressible Navier-Stokes equations were numerically solved. Blood was assumed to be Newtonian fluid and flows were treated as laminar. The cell-centred finite volume approach was used for spatial discretisation of the governing equations. Unstructured grid systems were employed so that the complicated geometry of each model could be properly expressed. The number of computational cells depended on the model: 886893 for Model 1, 1127077 for Model 2 and 1220816 for Model 3. Most computational cells were tetrahedra. Also hexahedra and trigonal prisms were allocated along the wall surfaces so that deterioration in accuracy in the boundary layers could be avoided. The SIMPLE algorithm was used for coupling of pressure and velocity.

The Navier-Stokes solver was parallelised by domain decomposition and implemented on a PC cluster with sixteen Pentium-4 processors.

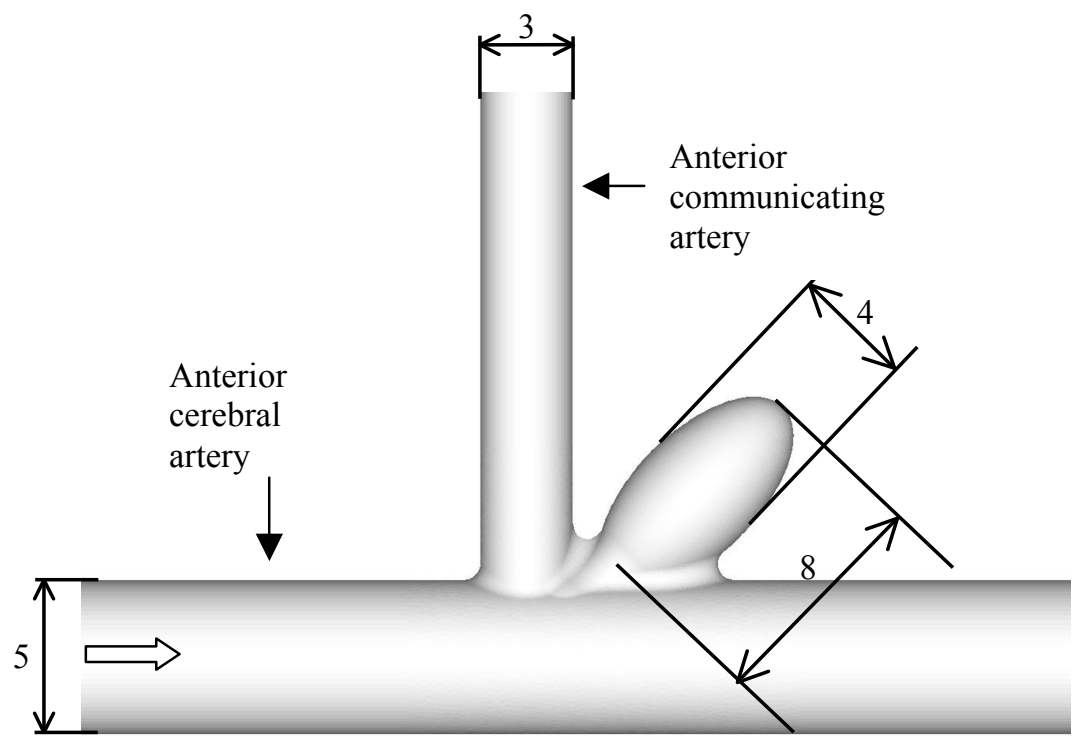

Figure 2: $\quad$ The geometry of Model 2 (all dimensions in $\mathrm{mm}$ ). 


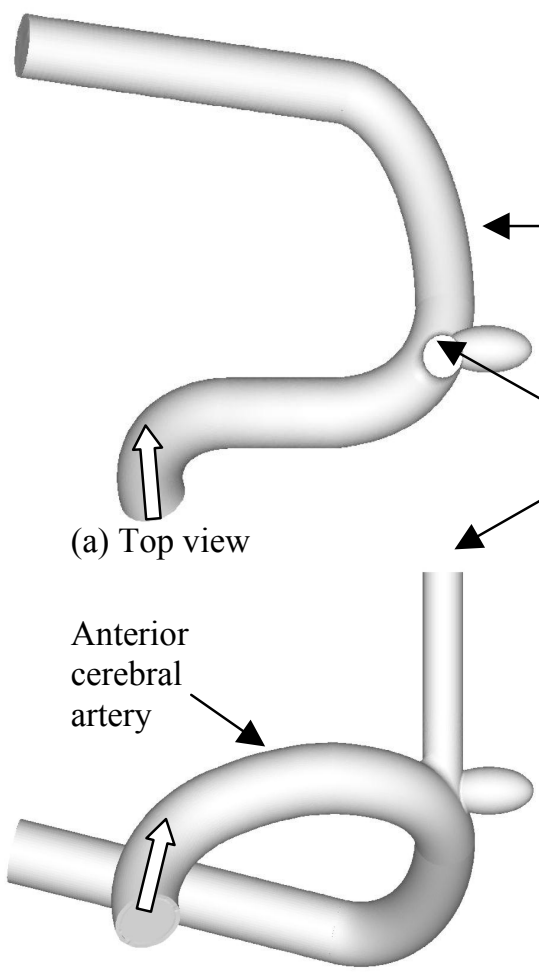

(b) Front view
Anterior cerebral artery

Figure 3: $\quad$ Views of Model 3.

\section{Results and discussions}

Figure 4 shows velocity vectors on the central cross-sectional plane in Model 1 at diastolic and systolic phases. It is clearly observed that the blood flow hit the edge of the aneurismal neck. Not only at the timings shown in fig.4, the blood flow was always impinging upon the neck at the anterior cerebral artery. This impingement is considered to cause haemolysis in which ADP is released from RBCs to the plasma. After the impingement upon the neck, some blood entered the dome with changing its direction and, again, hit the internal wall of the aneurysm. The spot of the second impingement depended on the time. At the higher flow rate (fig.4(b)), stronger inertia made the flow proceed deeper.

After the second impingement, the flow spread 3-dimensionally with reducing its speed as clearly seen in fig.5. The shape of these curved path lines agreed with the vortex reported by Takahashi et al [3]. Consequently, a low-speed region of the blood was formed and occupied a large part in the dome (see the lower half of the dome in fig.4). Importantly, the blood motion in the dome after 
the second impingement was so slow that there was a fairly long time for platelets to aggregate. According to Shimano et al. [4], aggregation occurred most actively in this region. The blood moving slowly in the dome changed its direction to the exit and returned to the arteries. The most blood proceeded to the anterior communicating artery after discharged from the dome.

(a) Diastolic phase

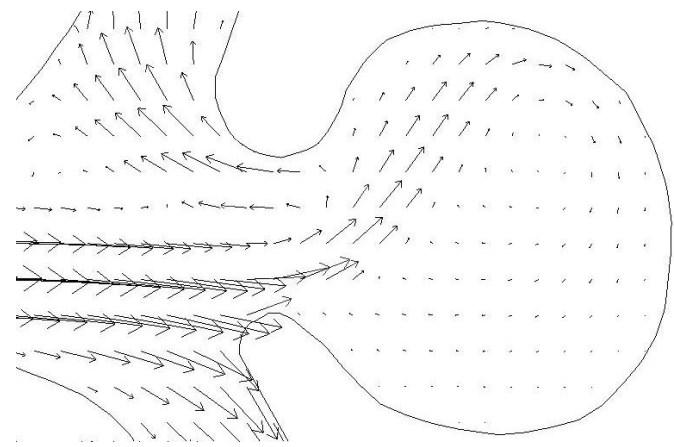

(b) Systolic phase

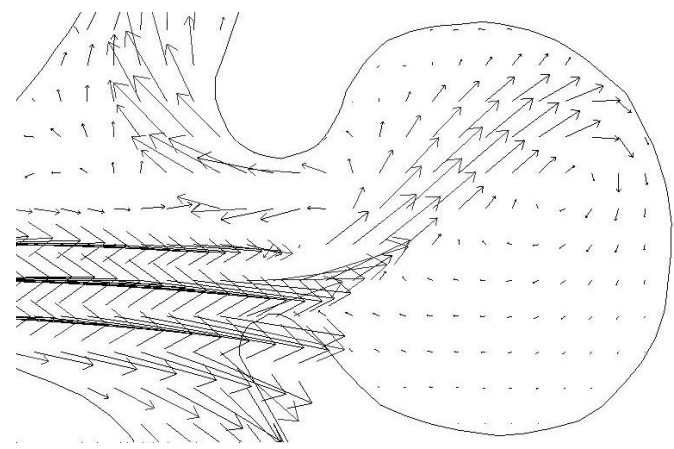

Figure 4: Velocity vectors on the central cross section in Model 1.

Figure 6 shows velocity vectors on the central plane in Model 2. The blood flow hit the right edge of the aneurismal neck at both timings. This feature is common to Models 1 and 2. However, in Model 2, the flow entered the dome along the aneurysmal wall and the second impingement, which was clearly seen in Model 1, was not present. The contrast between Models 1 and 2 can be highlighted if figs 5 and 7, in which particle paths in the domes are drawn, are compared. The fluid moving along the endothelium in Model 2 reached the depths of the dome, turned round and was discharged. This flow pattern in Model 2 agreed well with a video recorded in Shimamura's experiment [7]. The flow speed in the dome was so high that aggregation seems less likely than in Model 1. 


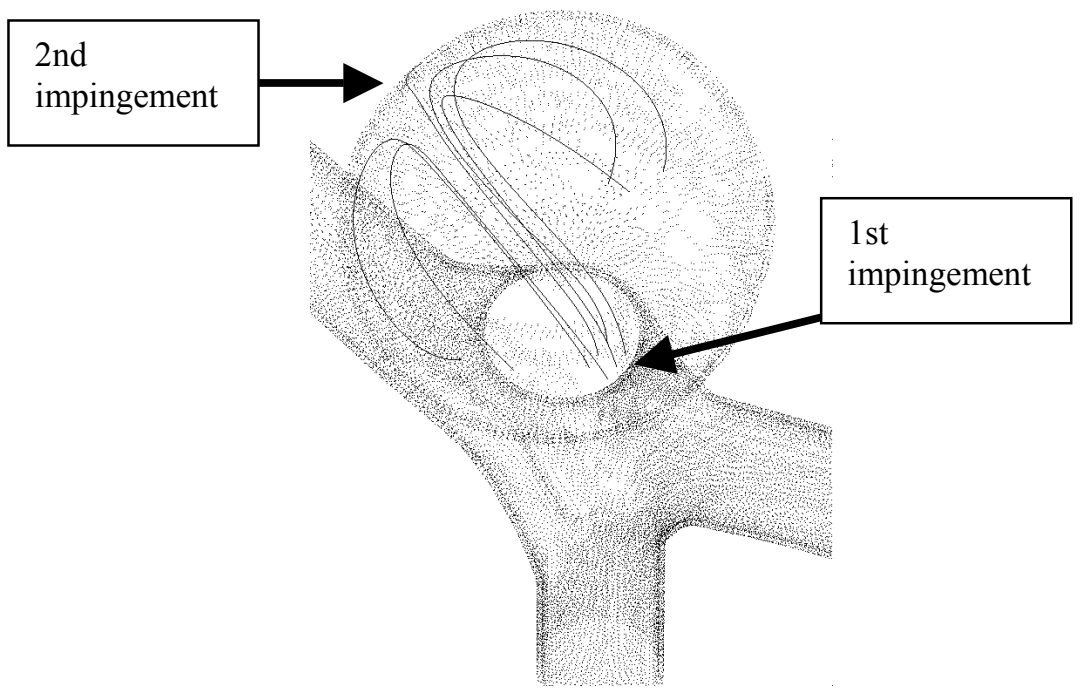

Figure 5: $\quad$ Particle paths after the first impingement to the aneurysmal neck (Model 1 around a diastolic phase).

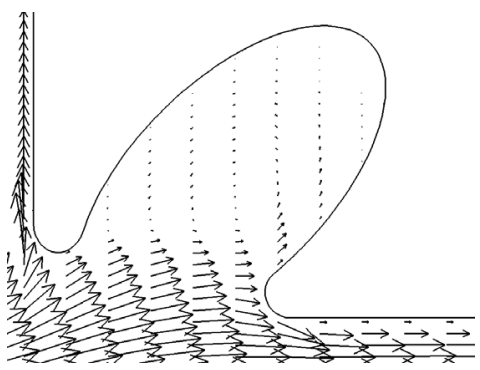

(a) Diastolic phase

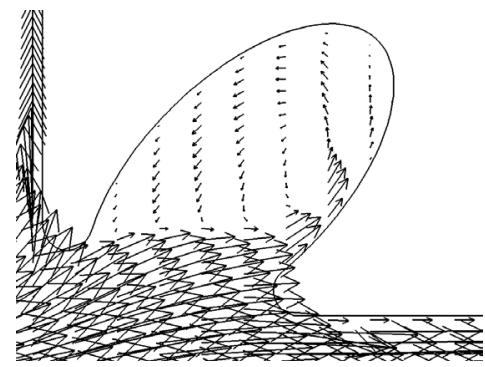

(b) Systolic phase

Figure 6: Velocity vectors on the central cross section in Model 2.

Calculated velocity vectors on two central planes of the aneurysmal dome in Model 3 are drawn in figs 8 and 9, of which directions of the view correspond to fig.1(a) and fig.1(b), respectively. In addition, the 3-dimensional structure of the flow in the aneurysmal dome is schematically illustrated in fig. 10. 
A strong impingement of the mainstream upon the aneurysmal neck was clearly observed in fig.8. As similar impingements were observed in all the models regardless of the bifurcation angles, impingements of this kind and resulting haemolysis are presumably common to most aneurysms at artery bifurcations.

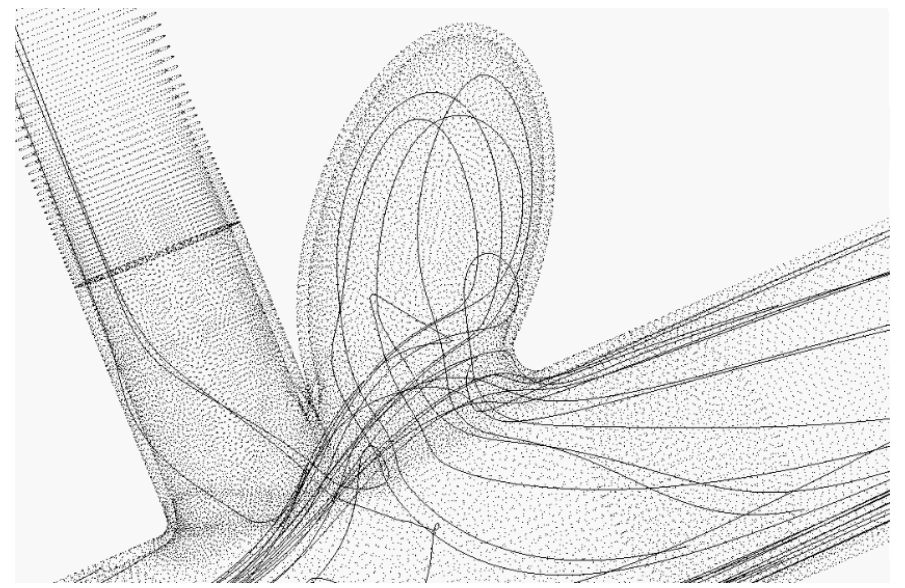

Figure 7: $\quad$ Particle paths in Model 2 around a systolic phase.

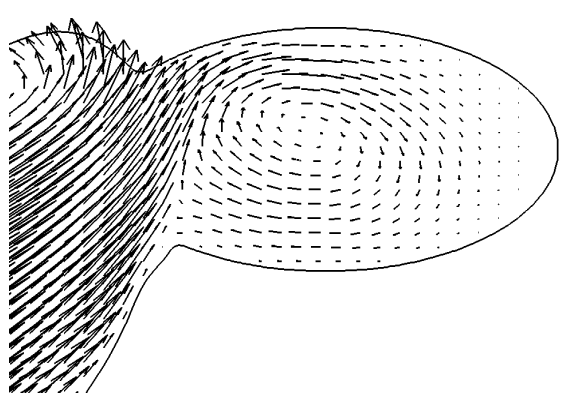

(a) Diastolic phase

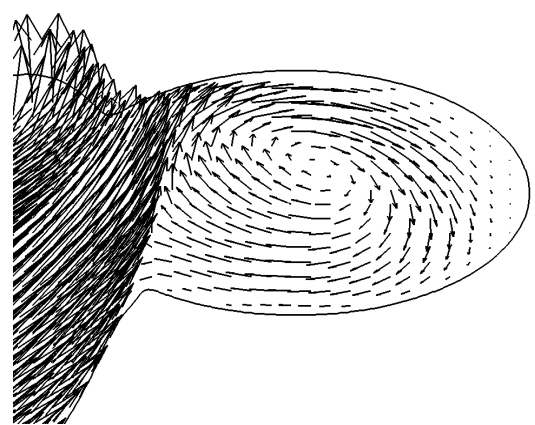

(b) Systolic phase

Figure 8: $\quad$ Velocity vectors on the central plane of the aneurysm in Model 3 parallel to fig.3(a). 


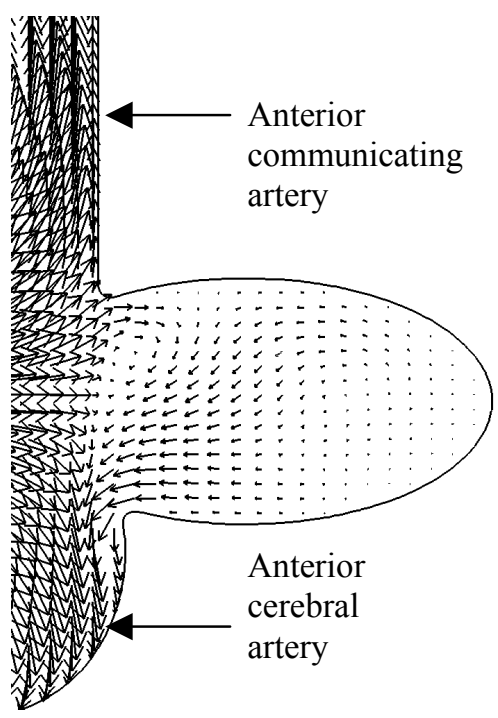

(a) Diastolic phase

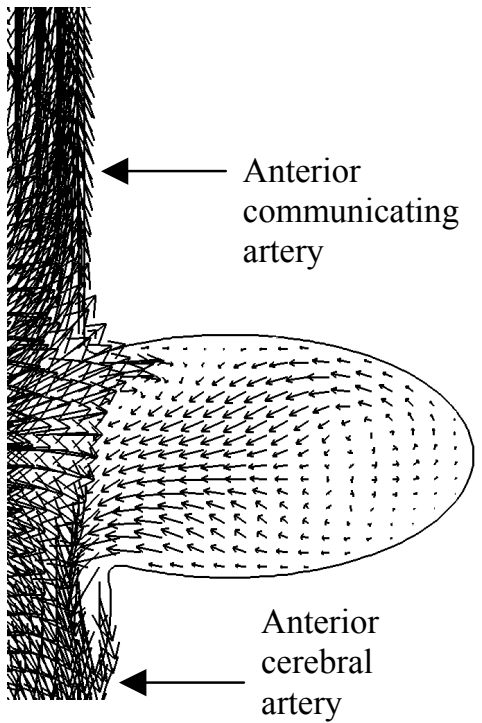

(b) Systolic phase

Figure 9: Velocity vectors on the cross-sectional plane parallel to fig.3(b) (Model 3).

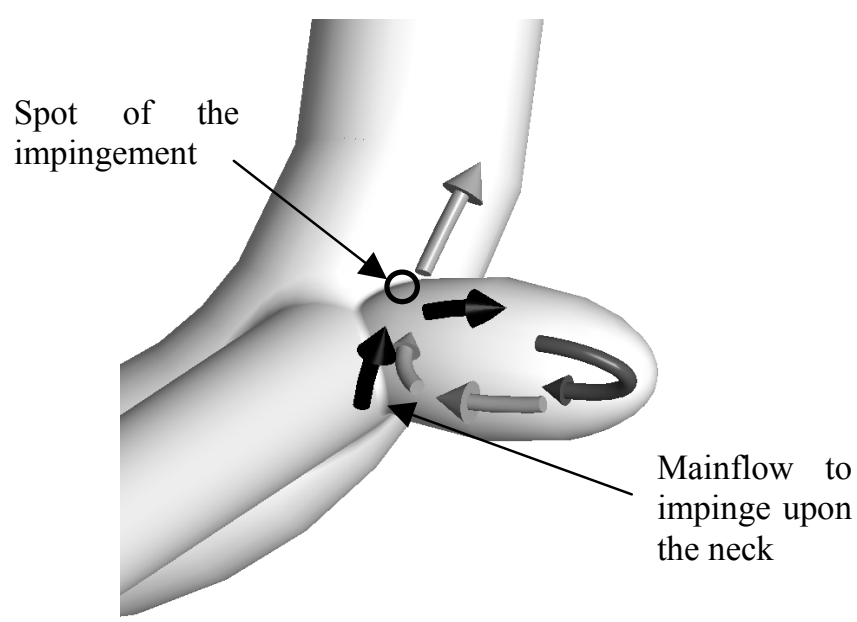

Figure 10: Schematic illustration of flow structure in the aneurysmal dome in Model 3. 
When Model 3 was used, the flow pattern in the aneurysmal dome was similar to that in Model 2 because, after the impingement upon the neck, the flow entered the dome along the endothelium and turned round. Unlike Model 1, neither impingement upon the internal aneurysmal wall nor extremely low speed region was observed in Model 3. According to Figure 9, the fluid was mainly discharged from the dome through a lower part of the neck and moved towards the anterior cerebral artery. As a whole, the flow structure in Model 3 was similar to that in Model 2 although the flow in Model 3 was more 3-dimensional.

The flow pattern in Model 1 was characterised by the second impingement within the aneurysm and the low-speed region. On the other hand, impingement upon the internal aneurysmal wall and an extremely low speed region were absent in Models 2 and 3. It can be concluded that platelet aggregation would have occurred less actively in Models 2 and 3 because ADP arising from the flow impingement upon the neck would have been swept out more quickly. The Y-shaped bifurcation or the angle of the aneurysmal dome does not seem responsible for the unique flow pattern in Model 1 because Models 2 and 3 showed similar flow features in spite of different angles of the bifurcation and dome protrusion. The aspect ratio (2.39 for Model 1 and approx. 2 for Models 2 and 3) might have had an influence on the different flow patterns to some extent. However, the larger volume of the aneurysmal dome in Model 1 seems a more reasonable explanation for the low speed region. The amount of fluid filling the dome was so large in Model 1 that the mainstream could not provide the fluid with enough energy to allow rapid motion in the dome. Therefore, the degree of platelet aggregation is presumably influenced not only by the aspect ratio but also the relative size of the aneurysmal dome to the artery.

\section{Conclusions}

Unsteady flow simulation was conducted for three different model aneurysms: Model 1 at a Y-shaped bifurcation, Model 2 at a T-shaped bifurcation and Model 3 which represented more realistic anatomical features of arteries. Impingements of the main flow upon the aneurysmal neck were observed in all the models. This means that haemolysis is likely to occur at the entries to most cerebral aneurysms. A large region filled with low speed fluid was found in Model 1 while fluid entering the domes was discharged quickly in Models 2 and 3 . Therefore, it can be concluded that platelet aggregation would have been less active in Models 2 and 3. This difference in flow patterns in the aneurysms was presumably due to the relative size of the aneurysmal dome to the artery diameter. The authors suggest that, in addition to the aspect ratio, the relative size of the aneurysm should be taken into consideration when the likelihood of platelet aggregation and thrombogenesis and the probability of rupture are discussed. 


\section{References}

[1] Ujiie, H., Tamano, Y., Sasaki, K. \& Hori, T., Is the aspect ratio a reliable index for predicting the rupture of a saccular aneurysm? Neurosurgery, 48(3), pp.495-503, 2001.

[2] Ujiie, H., Tachibana, H., Hiramatsu, O., Hazel, A.L., Matsumoto, T., Ogasawara, Y., Nakajima, H., Hori, T., Takakura, K. \& Kajiya, F., Effects of size and shape (aspect ratio) on the hemodynamics of saccular aneurysms: a possible index for surgical treatment of intracranial aneurysms, Neurosurgery, 45(1), pp.110-130, 1999.

[3] Takahashi, N., Ujiie, H., Yotoriyama, T., Suzuki, Y., Hori, T. \& Kaibara, M., Flow visualization study of the endothelialized glass aneurysm model implanting canine carotid artery (in Japanese with English abs.), J. Jpn. Soc. Biorheol., 18(4), pp.143-148, 2004.

[4] Shimano, K., Hayashi, T., Ujiie, H., Ono, T. \& Enomoto, Y., Modelling of platelet aggregation in aneurysm, Proc. of 7 th Int. Conf. On Modelling In Medicine and Biology, WIT Press, Southampton, pp.43-52, 2007.

[5] Torii, R., Oshima, M., Kobayashi, T., Takagi, K. \& Tezduyar, T.E., Influence of Wall Elasticity on Image-Based Blood Flow Simulations (in Japanese with English abs.), Transactions of Japan Society of Mechanical Engineers, Series A, 70(697), pp.1224-1231, 2004.

[6] Funazaki, K., Higashi, M., Yamada, K., Taniguchi, H. \& Tomura, N., FlowStructure Coupled Analysis of Cerebrovascular Artery with an Aneurysm of Realistic Geometry (in Japanese with English abs.), Transactions of Japan Society of Mechanical Engineers, Series B, 73(731), pp.1472-1479, 2007.

[7] Shimamura, T., A study on flow in aneurysms (in Japanese), Graduation thesis, Tokyo Science University, Tokyo, 2006.

[8] Satoh, A., Nakamura, H., Kobayashi, S., Miyata, A., Tokunaga, H., Wada, M. \& Watanabe, Y., Surgical approaches and techniques for anterior communicating artery aneurysms: from angioanatomical point of view (in Japanese with English abs.), Surgery for Cerebral Stroke, 30, pp.240-246, 2002. 